\title{
Enabling Single-Handed Interaction in Mobile and Wearable Computing
}

\author{
Hui-Shyong Yeo \\ University of St Andrews \\ Fife, Scotland, UK \\ hsy@st-andrews.ac.uk
}

\begin{abstract}
Mobile and wearable computing are increasingly pervasive as people carry and use personal devices in everyday life. Screen sizes of such devices are becoming larger and smaller to accommodate both intimate and practical uses. Some mobile device screens are becoming larger to accommodate new experiences (e.g., phablet, tablet, eReader), whereas screen sizes on wearable devices are becoming smaller to allow them to fit into more places (e.g., smartwatch, wrist-band and eye-wear). However, these trends are making it difficult to use such devices with only one hand due to their placement, limited thumb reach and the fat-finger problem. This is especially true as there are many occasions when a user's other hand is occupied (encumbered) or not available. This thesis work explores, creates and studies novel interaction techniques that enable effective single-hand usage on mobile and wearable devices, empowering users to achieve more with their smart devices when only one hand is available.
\end{abstract}

\section{Author Keywords}

Mobile devices, Wearable devices; Single-handed interaction; Subtle interaction; Smartwatch; Smart-ring; Commercial off-the-shelf;

\section{INTRODUCTION}

Some mobile devices such as smartphones and tablets are growing larger. Today, smartphones with a display size of over 5 and 6 inches are common. This makes single-handed usage problematic because it is difficult to reach all areas of the screen (Figure 1 left) due to the limited functional reach of the thumb [1]. This larger form factor also causes users to frequently change their hand grip and may contribute to people dropping the device accidentally. And while device manufacturers may intend for two handed use, an observation of over 1000 users, shows most users prefer to use one hand when using mobile devices [3].

Permission to make digital or hard copies of part or all of this work for personal or classroom use is granted without fee provided that copies are not made or distributed for profit or commercial advantage and that copies bear this notice and the full citation on the first page. Copyrights for third-party components of this work must be honored. For all other uses, contact the owner/author(s).

UIST'18 Adjunct, October 14-17, 2018, Berlin, Germany.

Copyright is held by the author/owner(s)

ACM ISBN 978-1-4503-5949-8/18/10.

http://dx.doi.org/10.1145/3266037.3266129

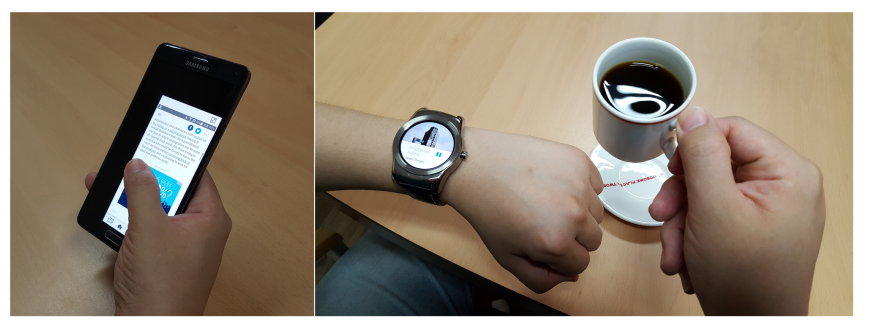

Figure 1. Example problems with single-handed interaction (left) it is difficult to reach all area of the screen with the thumb, resulting in alternative solution such as shrinking the user interface (right) it is difficult to impossible to touch the smartwatch for interaction when the second hand is occupied.

By contrast, wearable devices such as a smartwatch or smart eye-wear have very small touch surfaces for input. Therefore, these devices suffer from occlusion and the fat-finger [8] problem. In addition, interacting with a smartwatch typically requires two hands (Figure 1 right) - one hand (usually the non-dominant hand) is wearing it and providing support while the second hand (usually the dominant hand) interacts with the device by touching or moving the watch face.

This problem is exacerbated when the other hand is occupied (e.g., encumbered [7] when holding a drink) or not available (e.g., missing fingers/limbs or control issues). To address this we see the use of voice command or arm motion gestures but these can be less robust or perceived as awkward in public. This Single-Handed Interaction Problem (SHIP) is not new and has been studied before [4] for mobile devices. However, this problem has been evolving along with a growth of larger and smaller interaction surfaces. This $\mathrm{PhD}$ work explores the problems inherent in interaction when the screen size of a device gets larger or smaller and one-handed use is expected.

This general problem was first identified during the earliest stages of my PhD, when I was working on more expressive touch interaction with smartwatches $[10,12]$, as I quickly realized that two hands are required. As I am now approaching the end of the third year of my $\mathrm{PhD}$ program, I have published several related papers, with several projects on-going and several more planned for the future, which will be described next.

The UIST doctoral symposium provides an opportunity for feedback and advice, both for the remaining time of my PhD 

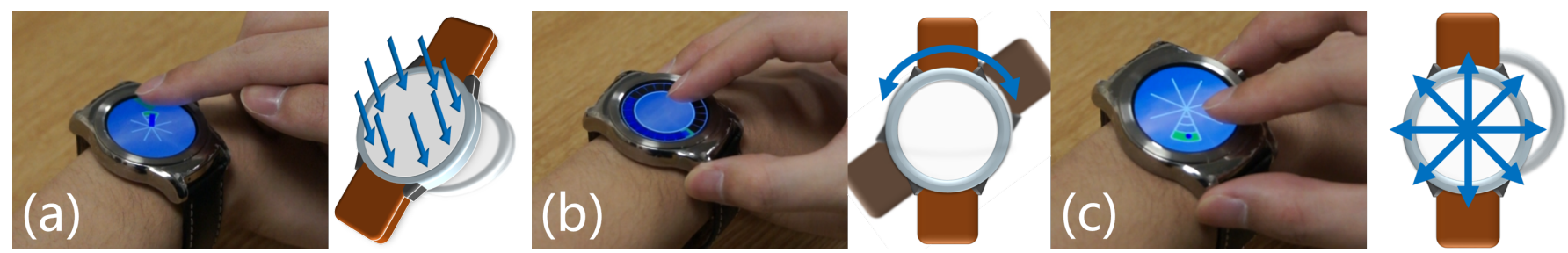

Figure 2. WatchMI supports pressure touch, twisting and panning gestures on unmodified smartwatch using built-in IMU without relying on additional sensors.

study and also for shaping the future direction for post $\mathrm{PhD}$ career.

\section{RESEARCH GOALS AND APPROACH}

My goal is to advance new approaches to address the singlehanded problem on both mobile and wearable devices, with a focus on commodity devices without or with minimal modification. Therefore the research questions are: i) How to create new sensing and input techniques for single-handed interaction based on off-the-shelf hardware and software? ii) How to support subtle and discreet interaction when using the technique so that it is less awkward? iii) How to develop interaction design patterns which are self-explanatory and can be adopted by others to improve "walk-up-and-use"?

\section{RESEARCH TO DATE}

\section{WatchMI, SideTap and Slingshot}

Initially, I was working on novel input methods for wearable devices using only built-in sensors. For example, WatchMI [12] supports pressure touch, twist and panning gesture (Figure 2) on commodity smartwatch without requiring pressure sensors whereas SideTap and Slingshot [11] support force tapping and hold and quick release gestures. While these techniques expand the input vocabulary of the device, they still require both hands for input. Hence, these have motivated the SHIP topic I pursue as my dissertation focus.

\section{RadarCat and SpeCam}

In both RadarCat [9] and SpeCam [10], I started exploring new ways to use a device with simple one-handed gestures, building on the idea of placement-aware computing [2]. By using a miniature radar sensor (RadarCat) or the front-facing camera of smartphone (SpeCam), the device can then recognize the surface where it is placed or touched. Thus, it enables singlehanded interaction such that placing the device on different surfaces or body parts can trigger shortcut commands. For example, it can launch different applications and switch mode based on which body part is touched, or pick a different color for a paint application (Figure 3).

\section{Shape Writing in Motion}

While the aforementioned placement aware interaction is intuitive, it is rather simplistic and limited to a few predefined commands. Next, I started exploring another common task that users perform on their device - text entry. In Shape Writing in Motion (SWiM) [13], I propose and evaluate a novel design point around a tilt-based text entry technique which

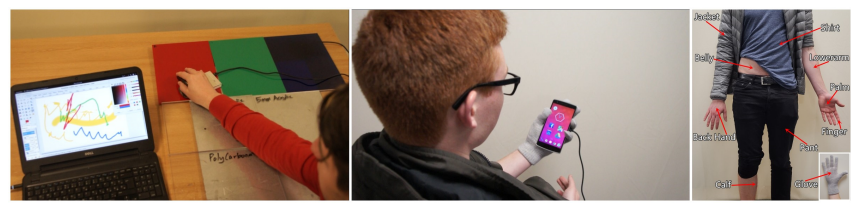

Figure 3. RadarCat applications: (left) color picker paint application (right) body shortcut by touching different body part with the device.

supports single handed usage on both large and small devices. The technique is based on the gesture keyboard (shape writing [5]). However, instead of drawing gestures with a finger or stylus, users articulate a gesture by tilting the device (Figure 5) to control a cursor.

The study results show that the technique has high immediate efficacy and is reasonably fast. Novice users achieve an entry rate of 15 words-per-minute (wpm) after minimal practice. A pilot longitudinal study reveals that a single participant achieved an entry rate of $32 \mathrm{wpm}$ after approximate 90 minutes of practice. SWiM is not meant to replace single-handed or two-handed typing in all situations, but rather to facilitate text entry when only one hand is available and when using a large device (e.g., phablet) or small device (e.g., smartwatch).

\section{Itchy Nose}

Besides voice input, a common method to interact with smart eye-wear (e.g., Google Glass) is to press the hardware buttons or use the touchpad. These actions may be considered disruptive, intrusive or socially unacceptable (i.e., distracting or rude) when socializing with others.

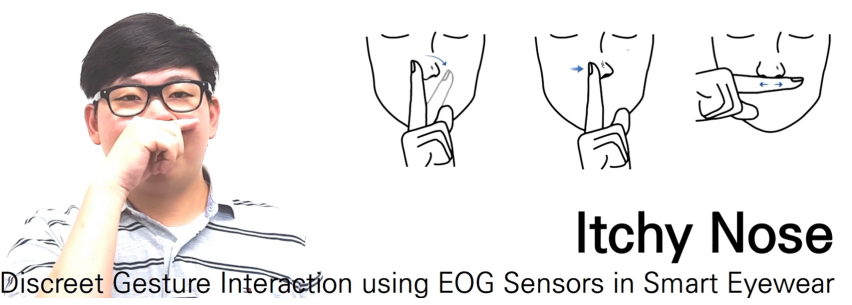

Figure 4. Itchy Nose uses finger movements on the nose to command a wearable computer. Itchy Nose may allow users to respond to notifications quickly without distracting nearby colleagues.

I propose a sensing technique for detecting finger movements on the nose, using EOG sensors embedded in the frame of a pair of eyeglasses. Eyeglass wearers can use their fingers to exert different types of movement on the nose, such as flicking, 

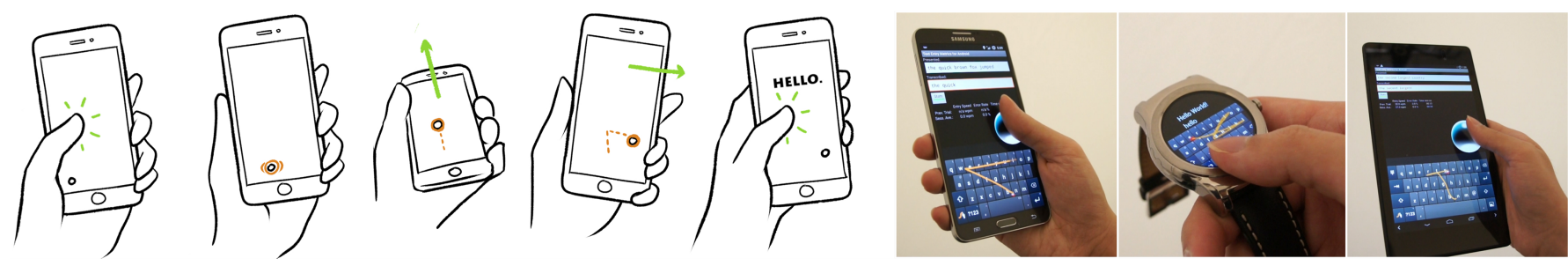

Figure 5. (left) SWiM technique: Tap and tilt the device to trace the word. (right) SWiM prototype on a phone, smartwatch and tablet.

pushing or rubbing, with only one hand (Figure 4). These subtle gestures can be used to control a wearable computer without calling attention to the user in public [6].

\section{ONGOING RESEARCH}

\section{LensWatch}

In LensWatch, I am exploring a new interaction technique that allows users to perform gestures and 2D targeting on arbitrary surfaces using their hand, much like using a computer mouse. Indeed, the hardware is comprised of an optical sensor attached to the bottom of a smartwatch, similar to those used in a computer mouse (Figure 6). Beside supporting single-handed interaction, it also supports "anywhere" interaction, including employing the users' own body parts as the surface. Therefore, it is less prone to movement error, e.g., while walking or jogging, whereas typical midair gesture systems that rely on arm motion gestures detected by inertial measurement unit (IMU) will suffer.

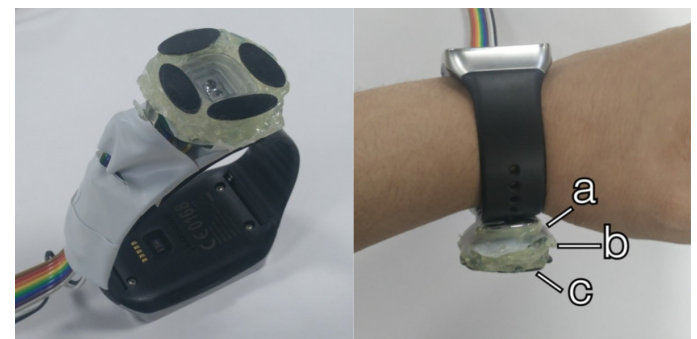

Figure 6. LensWatch allows gesture interaction on any surface, using an optical sensor mounted below the smartwatch.

A pilot study was performed in order to measure the performance of the device for both interactions on four different surfaces. After testing on these surfaces, the results show that the device presents an average error rate of $52 \%$ when performing gestures. Furthermore, for 2D targeting interaction, the device shows an average movement time of $3000 \mathrm{~ms}$, an average error rate of $17.2 \%$, and an average throughput of 0.85 in relation to Fitts's law. Currently, I am working on improving the system accuracy and create a newer version of the hardware that is smaller and fully wireless so it does not require tethered to the computer using cable.

\section{WRIST}

In Watch-Ring Interaction Sensing Techniques (WRIST), I am exploring a range of single-handed gestures which rely on simple wrist motion that is independent from the forearm motion, as the human wrist is one of the most dexterous parts of the human body. I propose a sensor fusion approach which combines IMU data from a smartwatch and a smart-ring. By measuring the relative difference in orientations, I extract a measure of the wrist angle that is position and orientation invariant. Employing the test hardware, I aim to demonstrate this is accurate, robust and enables a number of new and simple interactions. I demonstrate five applications to explore the potential of single-handed WRIST interaction in different usage scenarios i) gesture shortcuts, ii) 2D map navigation and iii) an analog clock iv) cursor control and v) gaming.

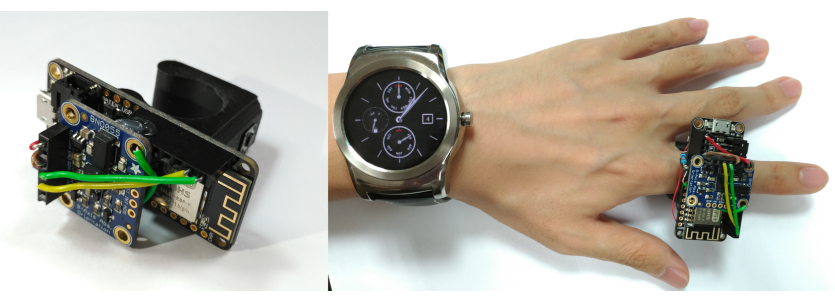

Figure 7. WRIST hardware consists of a customized smart-ring and a commodity smartwatch.

A preliminary study shows that this approach affords wrist gesture recognition even when the hand is in different orientations (e.g., raised or lowered at one's side). The ability to detect the same gesture combined with different hand postures allows the user to explore different context-aware interactions.

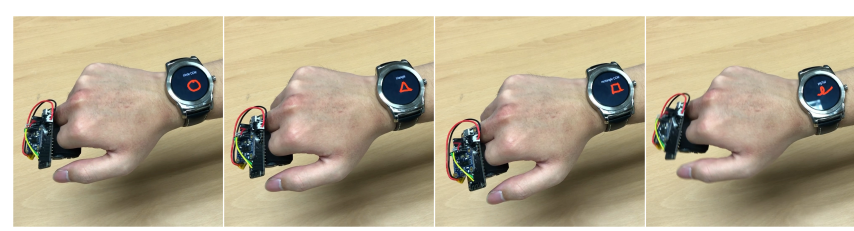

Figure 8. WRIST can recognize different wrist motions that are independent from the forearm movement, allowing more discreet and effortless gestures.

\section{REMAINING RESEARCH EFFORTS}

There are numerous extensions that I believe worth exploring next. First, while working on SWiM, I found that the SWiM technique has a greater potential that it can be extended to generic cursor control and pointing interaction, rather than limited to just text entry. I plan to conduct a study comparing this tilt-based cursor technique on the mobile device with other single-handed tilt-based techniques. 
Second, I am currently extending WRIST for both text entry and pointing interaction. WRIST could support interacting with virtual reality system and large displays. One of the envisioned advantage of WRIST pointing is that it supports dual precision pointing (macro and micro interaction) without requiring explicit mode switching or clutching. In addition, it could support two step interaction such as pie menu or marking menu. Watch and ring also can be worn on different hands to support bimanual interaction.

\section{DISSERTATION STATUS}

While I have advanced beyond the early stages of my $\mathrm{PhD}$ program and I am approaching the end of third year of my study, attending this doctoral symposium provides a excellent opportunity to help refine and shape my direction and focus. In the remaining period, I aim to complete and publish the ongoing projects and then submit my dissertation by the end of my fourth year.

\section{ACKNOWLEDGEMENTS}

I thank my advisor Professor Aaron Quigley and my mentor Nicolas Villar during my internship in Microsoft Research. My research is supported by SICSA and the University of St Andrews. I gratefully acknowledge the support of NVIDIA Corporation with the donation of the Titan X Pascal GPU used in my research.

\section{REFERENCES}

1. Joanna Bergstrom-Lehtovirta and Antti Oulasvirta. 2014. Modeling the Functional Area of the Thumb on Mobile Touchscreen Surfaces. In Proceedings of the 32Nd Annual ACM Conference on Human Factors in Computing Systems (CHI '14). ACM, New York, NY, USA, 1991-2000. DOI :

http://dx.doi .org/10.1145/2556288.2557354

2. Chris Harrison and Scott E. Hudson. 2008. Lightweight Material Detection for Placement-aware Mobile Computing. In Proceedings of the 21st Annual ACM Symposium on User Interface Software and Technology (UIST '08). ACM, New York, NY, USA, 279-282. DOI : http://dx.doi.org/10.1145/1449715.1449761

3. Steven Hoober. 2013. How Do Users Really Hold Mobile Devices? (2013). http://www. uxmatters. com/mt/archives/ 2013/02/how-do-users-really-hold-mobile-devices.php.

4. Amy K Karlson, Benjamin B Bederson, and Jose L Contreras-Vidal. 2008. Understanding one-handed use of mobile devices. In Handbook of research on user interface design and evaluation for mobile technology. IGI Global, 86-101.

5. Per-Ola Kristensson and Shumin Zhai. 2004. SHARK2: A Large Vocabulary Shorthand Writing System for Pen-based Computers. In Proceedings of the 17th Annual ACM Symposium on User Interface Software and Technology (UIST '04). ACM, New York, NY, USA, 43-52. DOI : http://dx. doi .org/10.1145/1029632 .1029640

6. Juyoung Lee, Hui-Shyong Yeo, Murtaza Dhuliawala, Jedidiah Akano, Junichi Shimizu, Thad Starner, Aaron Quigley, Woontack Woo, and Kai Kunze. 2017. Itchy
Nose: Discreet Gesture Interaction Using EOG Sensors in Smart Eyewear. In Proceedings of the 2017 ACM International Symposium on Wearable Computers (ISWC '17). ACM, New York, NY, USA, 94-97. DOI : http://dx. doi .org/10.1145/3123021.3123060

7. Alexander Ng, Stephen A. Brewster, and John H. Williamson. 2014. Investigating the Effects of Encumbrance on One- and Two- Handed Interactions with Mobile Devices. In Proceedings of the SIGCHI Conference on Human Factors in Computing Systems (CHI '14). ACM, New York, NY, USA, 1981-1990. DOI: http://dx. doi .org/10.1145/2556288.2557312

8. Katie A. Siek, Yvonne Rogers, and Kay H. Connelly. 2005. Fat Finger Worries: How Older and Younger Users Physically Interact with PDAs. In Human-Computer Interaction - INTERACT 2005, Maria Francesca Costabile and Fabio Paternò (Eds.). Springer Berlin Heidelberg, Berlin, Heidelberg, 267-280.

9. Hui-Shyong Yeo, Gergely Flamich, Patrick Schrempf, David Harris-Birtill, and Aaron Quigley. 2016a. RadarCat: Radar Categorization for Input \& Interaction. In Proceedings of the 29th Annual Symposium on User Interface Software and Technology (UIST '16). ACM, New York, NY, USA, 833-841. DOI : http://dx.doi. org/10.1145/2984511.2984515

10. Hui-Shyong Yeo, Juyoung Lee, Andrea Bianchi, David Harris-Birtill, and Aaron Quigley. 2017a. SpeCam: Sensing Surface Color and Material with the Front-facing Camera of a Mobile Device. In Proceedings of the 19th International Conference on Human-Computer Interaction with Mobile Devices and Services (MobileHCI '17). ACM, New York, NY, USA, Article 25, 9 pages. DOI :

http://dx.doi.org/10.1145/3098279.3098541

11. Hui-Shyong Yeo, Juyoung Lee, Andrea Bianchi, and Aaron Quigley. 2016b. Sidetap \& Slingshot Gestures on Unmodified Smartwatches. In Proceedings of the 29th Annual Symposium on User Interface Software and Technology (UIST '16 Adjunct). ACM, New York, NY, USA, 189-190. DOI : http://dx.doi.org/10.1145/2984751.2984763

12. Hui-Shyong Yeo, Juyoung Lee, Andrea Bianchi, and Aaron Quigley. 2016c. WatchMI: Pressure Touch, Twist and Pan Gesture Input on Unmodified Smartwatches. In Proceedings of the 18th International Conference on Human-Computer Interaction with Mobile Devices and Services (MobileHCI '16). ACM, New York, NY, USA, 394-399. DOI :

http://dx.doi .org/10.1145/2935334.2935375

13. Hui-Shyong Yeo, Xiao-Shen Phang, Steven J. Castellucci, Per Ola Kristensson, and Aaron Quigley. 2017b. Investigating Tilt-based Gesture Keyboard Entry for Single-Handed Text Entry on Large Devices. In Proceedings of the 2017 CHI Conference on Human Factors in Computing Systems (CHI '17). ACM, New York, NY, USA, 4194-4202. DOI : http://dx.doi.org/10.1145/3025453.3025520 